\title{
Experimental Investigation of High Frequency Plasma Oscillations within Hall Thrusters
}

\author{
A. Knoll", C. Thomas ${ }^{\dagger}$, N. Gascon ${ }^{\ddagger}$, M. Cappelli ${ }^{\S}$ \\ Stanford University, Stanford, California, 94305
}

\begin{abstract}
[Abstract] An experimental setup has been developed to measure high frequency plasma oscillations within the acceleration channel of a laboratory Hall thruster. The plasma oscillations are measured with three Langmuir probes separated by small axial and azimuthal offsets. This configuration permits the oscillations to be correlated with direction and wave number. This work is motivated by the anomalous electron transport phenomena, as plasma instabilities may play a crucial role in this transport process. Preliminary data has been gathered downstream of the exit plane of the thruster and suggests high frequency oscillations in the 1 to $10 \mathrm{MHz}$ range predominately in the axial direction. Work is currently underway to measure the high frequency oscillations within the acceleration channel at various axial locations.
\end{abstract}

\section{Introduction}

NE of the main obstacles to successful theoretical and numerical predictions of Hall thruster performance is to understand the physics governing the anomalous electron transport phenomenon within the acceleration channel. It has become common practice to deal with this anomalous transport using the Bohm conductivity model ${ }^{1}$. However, this has been shown to be experimentally inaccurate in work by Meezan et al. ${ }^{2}$ and Fife et al. ${ }^{3}$, particularly in the peak magnetic field region of the thruster.

There is experimental evidence that suggests that this anomalous transport is caused by high frequency plasma oscillations within the Hall thruster discharge ${ }^{2,4}$. The purpose of this work is to collect a base of experimental measurements of high frequency plasma oscillations within a laboratory Hall thruster. This data is being collected at various axial locations within the acceleration channel, and under several operating regimes of the thruster.

A Hall thruster numerical simulation formulated in the radial-axial plane has revealed high frequency plasma oscillations at the exit of the acceleration channel. It is likely that there are also oscillations at high frequencies in the azimuthal direction. However, the coordinate system of this model prohibits observation of these oscillation modes directly. It has been theorized that the axial perturbations may in fact be coupled to the azimuthal modes, and that these azimuthal waves enhance the transport of electrons. One of the key contributions of this work will be to observe the characteristics of the plasma oscillations that exist in the acceleration channel. Ultimately, numerical models will have to capture these same modes in order to reflect the physics involved in the transport process.

This work closely follows similar experimental studies performed by Guerrini et al. ${ }^{5}$ and Litvak et al. ${ }^{6}$. This study goes further by correlating the various wave modes with direction and wave number using three separate Langmuir probes. The thruster that is employed in these experimental trials is the Stanford Hall Thruster. This thruster has been used in many previous experiments, and its performance parameters have been documented in studies by Meezan et al. ${ }^{2}$ and Hargus et al. ${ }^{7}$. The Stanford Hall Thruster is a custom designed low power device with an acceleration channel $90 \mathrm{~mm}$ in diameter, $11 \mathrm{~mm}$ in width, and $80 \mathrm{~mm}$ in length.

\footnotetext{
* Research Assistant, Mechanical Engineering: Thermosciences Division, Building 520, AIAA Student

$\dagger$ Research Assistant, Mechanical Engineering: Thermosciences Division, Building 520, AIAA Student

‡ Postdoctoral Fellow, Mechanical Engineering: Thermosciences Division, Building 520, AIAA Member

$\S$ Professor, Mechanical Engineering: Thermosciences Division, Building 520, AIAA Member
}

1

American Institute of Aeronautics and Astronautics 


\section{Experimental Apparatus}

The experimental setup consists of three separate plasma probes, which are inserted into the acceleration channel through a slot in the side of the thruster. The probes are connected to a high frequency impedance matching circuit located within the vacuum chamber. A translating stage allows the probes to be moved along the axis of the thruster. A schematic diagram of the experimental setup is shown in Fig. 1.

The three probes are aligned along the axis and azimuth of the thruster as shown in Fig. 2. This configuration allows the spectral density of the oscillations to be correlated with velocity, angle, and frequency across the azimuthal-axial plane of the thruster ${ }^{8}$. The fluctuation of the current collected by the probes was measured in the ion saturation regime, thus measuring the local fluctuations in plasma density.

Previous experimental work by Litvak et al. ${ }^{6}$ has demonstrated the need for impedance matching electronics in order to resolve the high frequency current oscillations collected by the probes. The impedance matching technique used in this study was based on the circuit concept presented by Litvak et al. ${ }^{6}$. A schematic of

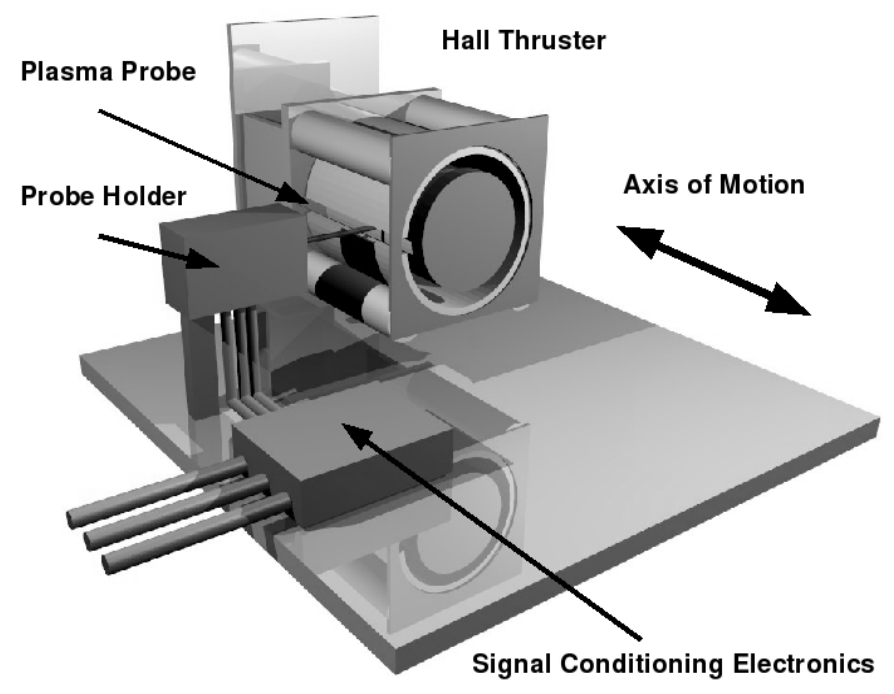
the circuit used in the current work is Figure 1. Experimental Setup. shown in Fig. 3. This basic circuit was used three times in parallel; once for each tip of the Langmuir probe. The heart of this circuit is a high frequency Operational Amplifier (OpAmp) that is decoupled from the plasma source by a 1:1 transformer. The output from the OpAmp is then matched to the coaxial lines transmitting this signal to the data acquisition equipment. The DC biasing circuit allows the probes to be biased to a specified potential. Ideally the amplification factor of this circuit should be constant. However, in practice the amplification factor varies with frequency. This issue was addressed by calibrating the gain as a function of frequency using a sinusoidal signal generator.

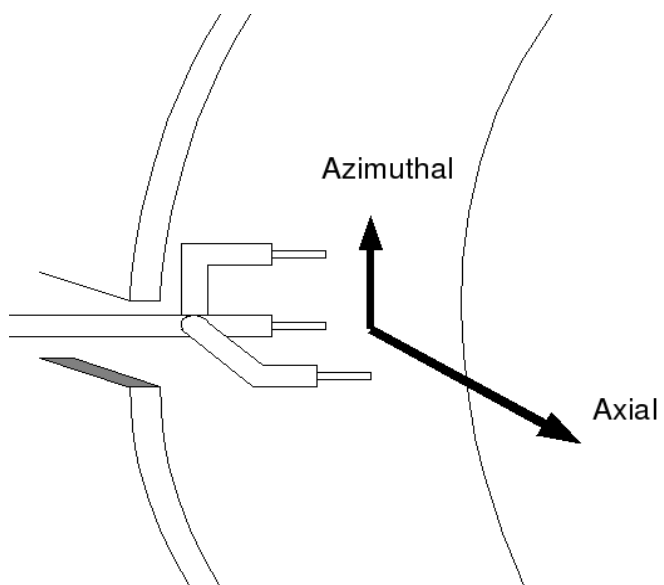

Figure 2. Probe Orientation within Discharge Channel. 


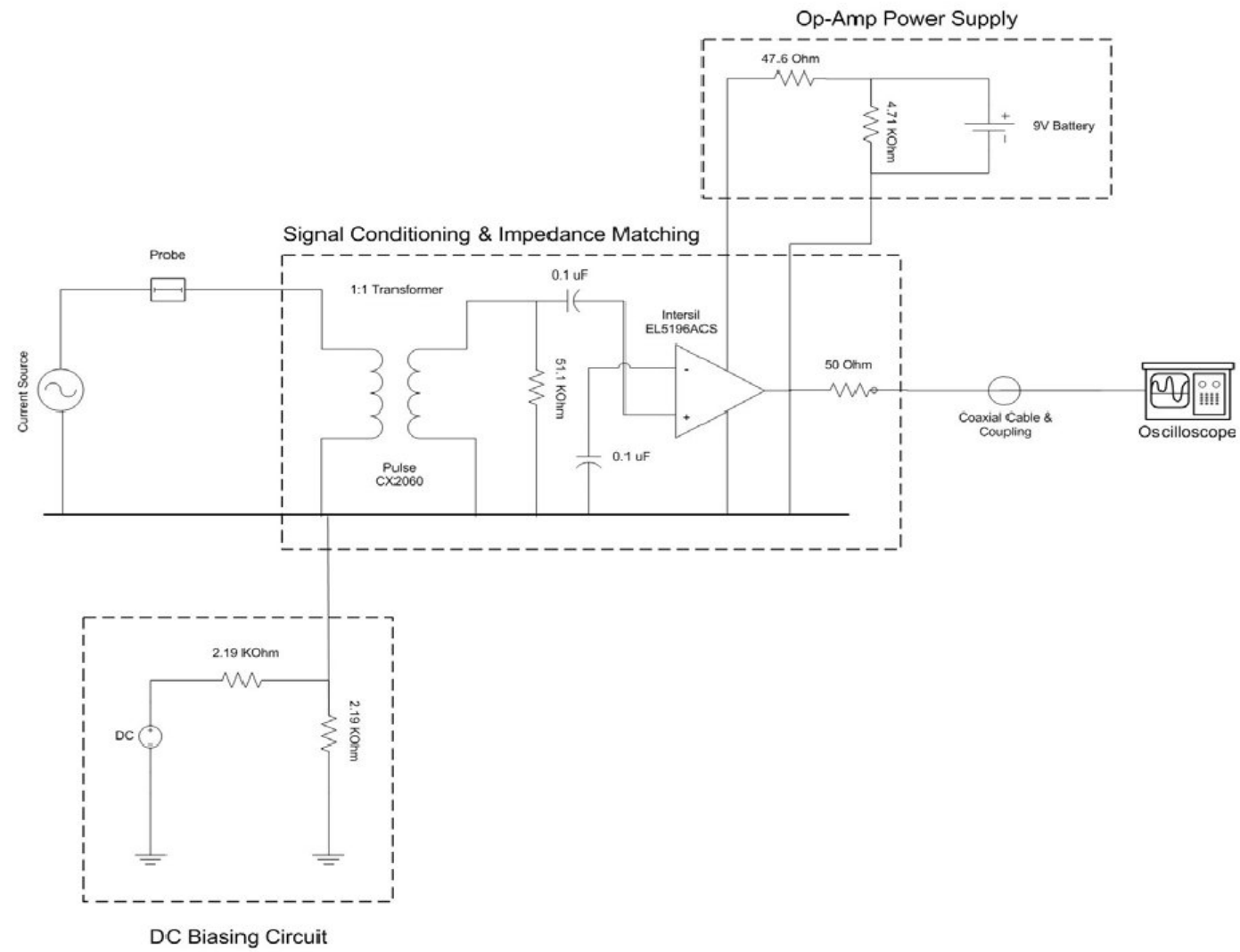

Figure 3. High Frequency Impedance Matching Circuit.

One of the challenges encountered during this project was appropriate thermal management of the electronic components within the impedance matching circuit. The OpAmps used in this circuit generated an excess amount energy when operated in vacuum conditions. To alleviate this problem a copper radiative dissipater was used as a case for the electronic components. A layer of felt alumina ceramic acted as a thermal conductor between the electronic components and the casing.

Another challenge that was encountered during the experimental trials was isolating the sensitive microelectronics from the plasma source. Due to current transients during the startup of the thruster, the OpAmps used in this circuit were damaged sporadically. This problem is being addressed by biasing the probes independently of the floating ground potential of the circuit; creating complete electrical isolation of the electronics from the plasma source.

\section{Data Reduction Methodology}

The data collected in this experiment consists of a set of discrete voltage measurements collected by three fixed probes operating in the ion saturation regime. These probes are separated by $1 \mathrm{~cm}$ offsets in the axial and azimuthal directions of the Hall thruster as indicated in Fig. 4. 


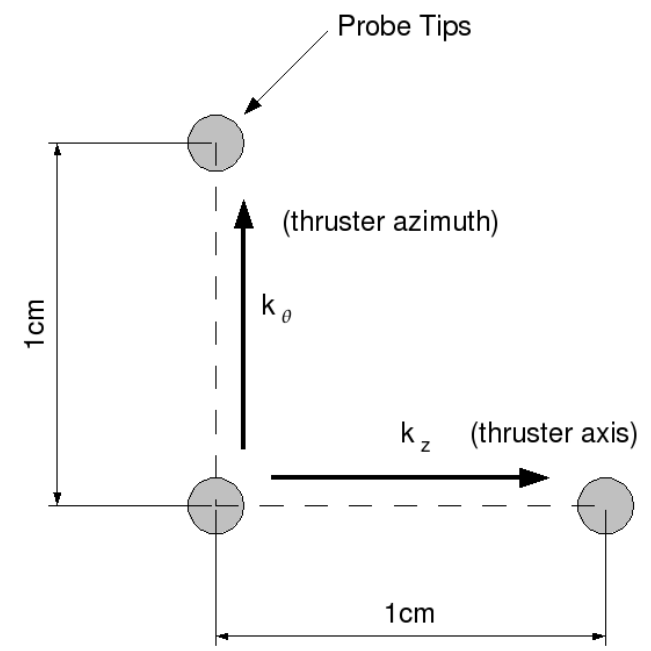

Figure 4. Schematic Probe Layout.

Appropriate analysis of the data permits the spectral density to be correlated with frequency $(\omega)$, wave number $(\mathrm{k})$, and direction $(\alpha)$ measured with respect to the axis of the thruster. Following the techniques introduced by Beall et al. ${ }^{9}$ the local spectrum between two probe pairs can be correlated with wave number by

$$
S(k, \omega)=<\frac{1}{2}\left(B^{*}(\vec{x}, \omega) B(\vec{x}, \omega)+B^{*}(\vec{x}+\vec{d}, \omega) B(\vec{x}+\vec{d}, \omega)\right) \delta\left(k_{d}-k\right)>
$$

Where $\mathrm{S}$ is the local spectrum, $\mathrm{B}$ is the signal under investigation, $\mathrm{d}$ is the separation vector between the probe pairs, and $\mathrm{x}$ is the probe positions. Similar to the work by Dudok de Wit et al. ${ }^{10}$, the Fourier transforms are replaced by Morlet wavelet transforms. This is done to increase the signal to noise ratio, and to eliminate the constraints of planarity, stationarity, and homogeneity ${ }^{10}$. The Morlet wavelet function is given by

$$
h(t)=\frac{1}{\pi^{1 / 4}} \exp (-2 \pi j t) \exp \left(-t^{2} / 2\right),
$$

and the corresponding wavelet transform is then

$$
B(\vec{x}, \omega)=\int B(\vec{x}, t) \frac{1}{\sqrt{a}} h^{*}\left(\frac{t-\tau}{a}\right) d t
$$

In this equation $k_{d}=\vec{k} \dot{\vec{d}} /|\vec{d}|$, which is calculated by relating the cross phase of two measurements. More information on this technique can be found in the reference by Dudok de Wit et al. ${ }^{10}$.

The method of fixed probe pairs is used to correlate the data with propagation angle as follows. At each frequency the azimuthal and axial wave number is calculated using only the two probe pair along its respective axis. These two wave numbers then form the components of the resultant wave at a given frequency, therefore establishing the orientation of the oscillation $(\alpha)$. 


\section{Experimental Results}

The experimental results presented below were collected $5 \mathrm{~cm}$ downstream of the acceleration channel exit. A detailed map of the wave modes within the acceleration channel is currently underway. The spectral power density of the observed oscillations as a function of frequency is shown in Fig. 5. These results were collected by the middle probe of the three probe setup. This data shows a visual similarity to similar high frequency measurements in studies by Guerrini et al. ${ }^{5}$ and Litvak et al. ${ }^{6}$. It should be pointed out that the impedance matching circuit has only been calibrated to $15 \mathrm{MHz}$, due to a limitation of the signal generator used for the calibration procedure. The circuit has a theoretical upper limit of $500 \mathrm{MHz}$, but results at higher frequencies are currently considered suspect. This is particularly true in the case of a spike in the spectral power density at $230 \mathrm{MHz}$. Further calibration tests are needed to determine if these features are physical, or purely an electrical anomaly within the impedance matching circuit.

The data has been correlated with wave number and direction using the techniques described above. The results of this analysis are shown in four plots below. These plots show the spectral power density as a function of frequency and wave number in the axial and azimuthal directions independently (Fig. 6 and 7). Also, two combined plots show the spectral power density as a function of frequency versus angle (Fig. 8) and angle versus wave number (Fig. 9). It can be observed from these plots that interesting features are visible at high frequencies around $10 \mathrm{MHz}$ and at a wave number of approximately $40 \mathrm{~m}^{-1}$, and that these features are primarily in the axial direction. Further investigation is needed to determine the nature of these modes, and their significance to the process of axial electron transport.

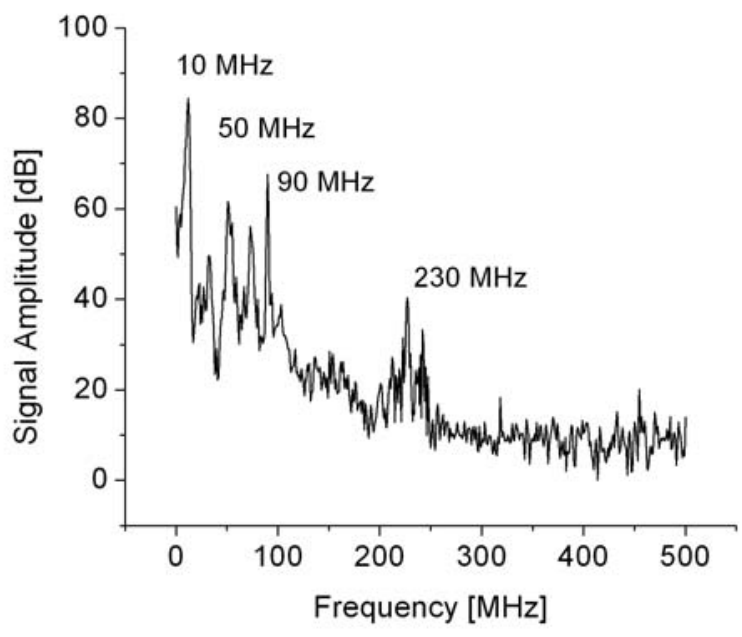

Figure 5. Frequency Spectra for Center Probe.

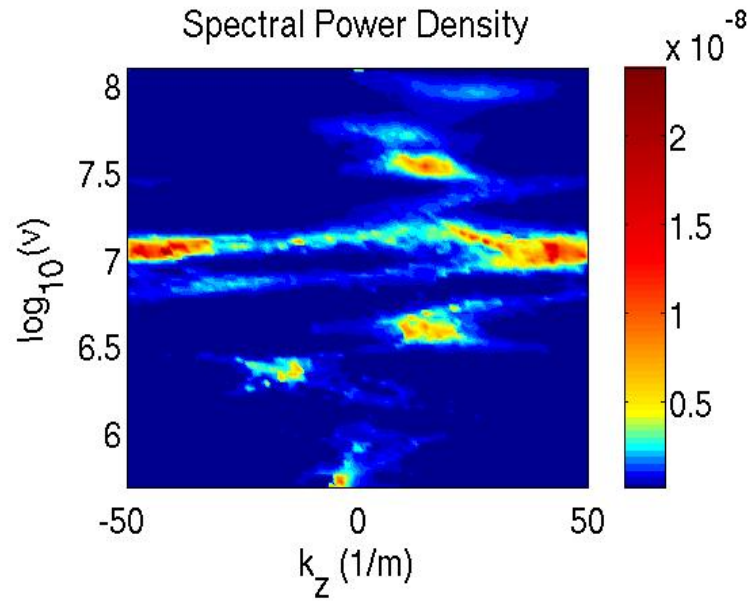

Figure 6. Frequency versus Axial Wave Number Spectral Density.

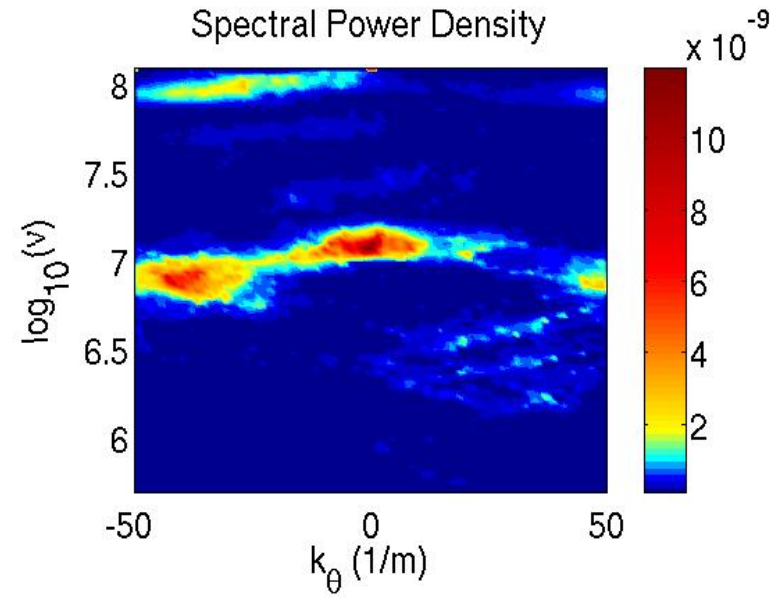

Figure 7. Frequency versus Azimuthal Wave Number Spectral Density. 

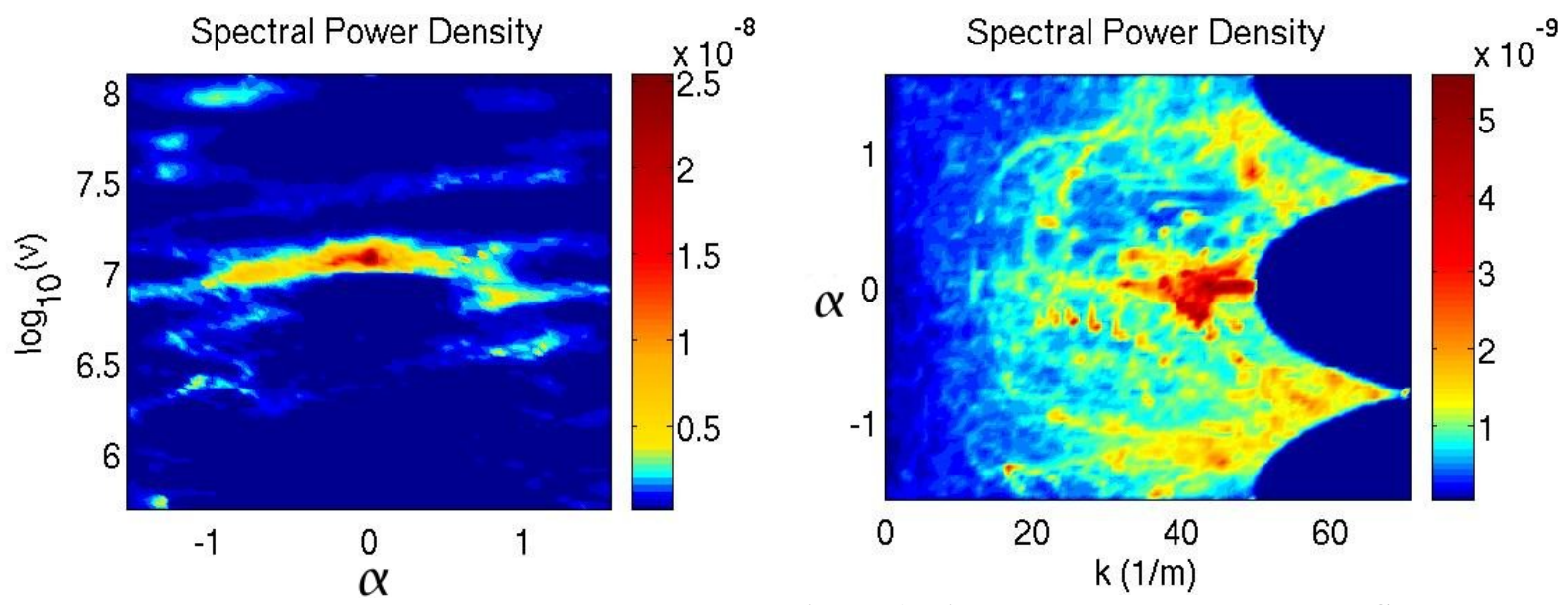

Figure 8. Frequency versus Angle Spectral Density. Figure 9. Angle versus Wave Number Spectral

Density.

\section{Conclusions}

An experimental setup has been developed to characterize the high frequency plasma oscillations within the acceleration channel of a laboratory Hall thruster. This setup permits the oscillations to be correlated with wave number and direction. Preliminary experimental observations have been gathered downstream of the acceleration channel exit. Analysis of this data has suggested wave modes in the range of 1 to $10 \mathrm{MHz}$ aligned mainly in the axial direction.

Work is currently underway to analyze instabilities in the acceleration channel of the thruster at various axial locations and under different operating regimes of the thruster. The aim of this work is to form a clear picture of the high frequency oscillation modes that exist within the Hall thruster; which may harbor a large variety of such waves in various orientations and wave lengths. The usefulness of this data falls into two categories. First, it can be used to directly study the contribution of these oscillations to the anomalous electron transport process. Second, it provides a wealth of experimental data that may be compared against numerical Hall thruster simulations. It may be vital to successfully reproduce these high frequency instabilities numerically in order to correctly capture the physics leading to the anomalous electron transport process.

\section{References}

${ }^{1}$ D. Bohm, in The Characteristics of Electrical Discharges in Magnetic Fields, edited by A. Guthrie and R. K. Wakerling, Chap. 2, p. 65., McGraw-Hill, New York, (1949)

${ }^{2}$ B. Meezan, W. A. Hargus, Jr., and M. A. Cappelli, "Anomalous electron mobility in a coaxial Hall discharge plasma", Physical Review E., Volume 63, 026410 (2001)

${ }^{3}$ J. M. Fife, Hybrid-PIC Modeling and Electrostatic Probe Survey of Hall Thrusters, Ph. D. Thesis: Massachusetts Institute of Technology, Department of Aeronautics and Astronautics, September (1998)

${ }^{4}$ G. C. Janes and R. S. Lowder, "Anomalous Electron Diffusion and Ion Acceleration in a Low-Density Plasma", The Physics of Fluids, Vol. 9, No. 7, June (1966)

${ }^{5} \mathrm{G}$. Guerrini and C. Michaut, "Characterization of high frequency oscillations in a small Hall-type thruster", Physics of Plasmas, Vol. 6, No. 1, January (1999)

${ }^{6}$ A. A. Litvak, Y. Raitses, and N. J. Fisch, "High-frequency Probing Diagnostic for Hall Current Plasma Thrusters", Review of Scientific Instruments, Vol. 73, No. 8, August (2002) 
${ }^{7}$ W. A. Hargus, Investigation of the plasma acceleration mechanism within a coaxial Hall thruster, Ph. D. Thesis: Stanford University, Department of Mechanical Engineering, (2001)

${ }^{8}$ N. Gascon and M. A. Cappelli, "Plasma instabilities in the ionization regime of a Hall thruster", Powered Flight - The Next Century, (2003)

${ }^{9}$ J. M. Beall, Y. C. Kim, and E. J. Powers, "Estimation of wavenumber and frequency spectra using fixed probe pairs", Journal of Applied Physics, Vol. 53, No. 6, June (1982)

${ }^{10}$ T. Dudok de Wit, V. V. Krasnosel'skikh, S. D. Bale, M. W. Dunlop, H. Luhr, S. J. Schwartz, and L. J. C. Woolliscroft, "Determination of dispersion relations in quazi-stationary plasma turbulence using dual satellite data", Geophysical Research Letters, Vol. 22, No. 19, October (1995) 